\title{
A newly identified IncRNA H1FX-AS1 targets DACT1 to inhibit cervical cancer via sponging miR-324-3p
}

Xinghua Shi ${ }^{1,2 \dagger}$, Jinzhi Huo ${ }^{3 \dagger}$, Xingping $\mathrm{GaO}^{4 \dagger}$, Hua Cai ${ }^{5^{*}}$ and Weipei Zhu ${ }^{1 *}$ (B)

\begin{abstract}
Background: Cervical cancer (CC) is the 4th most common cancer-related death in gynecological cancer worldwide. It has been reported that many IncRNAs contribute to oncogenesis although the fundamental mechanisms are basically unknown. Here, we aimed to identify a novel IncRNA H1FX-AS1 and explore a ceRNA network in CC oncogenesis and progression.

Methods: The expression level and the association with the prognosis of H1FX-AS1 in CC patients were analyzed based on Cancer Genome Atlas (TCGA) datasets, and further verified in 50 CC patients. The biological role of H1FXAS1 was investigated in vitro and in vivo by over-expression of H1FX-AS1 in CC cells; the potential binding sites between H1FX-AS1 and miRNA, between miR-324-3p and DACT1 were predicted by LncBASE and Targetscan respectively, which were further verified by dual-luciferase reporter assay, RNA pull-down and point mutation; the relationship between genes was analyzed by Pearson correlation; the rescue experiments were used to further explore the involved molecular mechanism.
\end{abstract}

Results: Lower H1FX-AS1 expression in CC tissues was found to be associated with the poor prognosis of CC patients. Over-expression of H1FX-AS1 inhibited CC cell proliferation, migration and invasion, while induced apoptosis by sponging miR-324-3p to up-regulate the DACT1 expression level.

Conclusion: A novel IncRNA H1FX-AS1 was identified, which acted as a competing endogenous RNA (ceRNA) of miR-324-3p to inhibit DACT1 mediated CC progression. Therefore, H1FX-AS1 is a new prognostic predictor and targeting the factors in the H1FX-AS1/miR-324-3p/DACT1 axis is the novel potential therapeutic strategy for CC.

Keywords: Cervical cancer, H1FX-AS1, miR-324-3p, DACT1, ceRNA

*Correspondence: ch17762085512@sina.com; qinghaixi@21cn.com ${ }^{+}$Xinghua Shi, Jinzhi Huo and Xingping Gao contributed equally to this work

${ }^{1}$ Department of Obstetrics and Gynecology, the Second Affiliated Hospital of Soochow University, No. 1055 Sanxiang Road, Suzhou 215000,

China

${ }^{5}$ Department of Gynecology, Maternal and Child Health

Hospital of Liaocheng, No. 56 Changjiang Road, High-tech Zone, Liaocheng 252000, China

Full list of author information is available at the end of the article

\section{Background}

The cervical cancer (CC) ranks the 4th most frequently diagnosed cancer and the 4th leading cancer-related death in the females with a predictable 311,000 deaths and 570,000 cases in 2018 worldwide, although the screening and HPV vaccine have been applied; meanwhile, CC ranks the second in the mortality and incidence following the breast cancer in the lower Human Development Index settings, the most usually diagnosed malignancy in 28 countries and the leading cancerrelated death in 42 countries [1], which rise severe public

(c) The Author(s) 2020. This article is licensed under a Creative Commons Attribution 4.0 International License, which permits use, sharing, adaptation, distribution and reproduction in any medium or format, as long as you give appropriate credit to the original author(s) and the source, provide a link to the Creative Commons licence, and indicate if changes were made. The images or other third party material in this article are included in the article's Creative Commons licence, unless indicated otherwise in a credit line to the material. If material is not included in the article's Creative Commons licence and your intended use is not permitted by statutory regulation or exceeds the permitted use, you will need to obtain permission directly from the copyright holder. To view a copy of this licence, visit http://creativeco mmons.org/licenses/by/4.0/. The Creative Commons Public Domain Dedication waiver (http://creativecommons.org/publicdomain/ zero/1.0/) applies to the data made available in this article, unless otherwise stated in a credit line to the data. 
issues in woman health. Moreover, despite the advances in diagnosis and the aggressive treatment, the prognosis of CC is still quite unsatisfactory with a 5-year overall survival under $40 \%$ in nearly all countries, due to the cancer metastasis, recurrence, and unclear pathogenesis [2]. Therefore, it is critical to explore the novel molecular mechanism of CC oncogenesis, and to develop the new biomarkers for the diagnosis, prevention, treatment and prognosis of CC patients.

Non-coding RNAs (ncRNAs), including microRNA (miRNA) and long non-coding RNA (lncRNA), have been recently reported to be the important novel biomarkers in oncogenesis [3, 4]. LncRNAs include many transcript types with a minimum of 200 nucleotides (nt) without protein-coding ability [5-7]. The location relationship of lncRNAs with other genes includes introns, intergenic gene or overlapping in an antisense orientation, while the lncRNA expression is usually tissue specific, involving in diverse genetic processes, for example, the mRNA stability, transcription and posttranslational modification [8-10], thus contributes to the oncogenesis and tumor progression [11]. Competing endogenous RNA (ceRNA) has been noted to be a new regulatory mechanism model and recognized to mediate the lncRNA function. In which, the expression levels of both mRNA and lncRNA are regulated by competitively binding to the shared miRNAs and degradation of the target mRNAs [12].

By searching the literatures we found the function of H1FX-AS1 in CC was not reported. According to the TCGA datasets we identified that H1FX-AS1 was downregulated in the $\mathrm{CC}$ tissues, which was confirmed to be associated with the overall survival in CC patients after our further analysis and confirmed the identical expression level in our pretest experiments. By the bioinformatics prediction, miR-324-3p was designated as the potential target of H1FX-AS1, which could potentially bind to DACT1, therefore, miR-324-3p was investigated as a prospective ceRNA of H1FX-AS1, and the potential of H1FX-AS1/miR-324-3p/DACT1 ceRNA network in $\mathrm{CC}$ progression as the novel prognostic predictors, the prevention and therapeutic targets for the $\mathrm{CC}$ patients was investigated in our current work.

\section{Patients and methods}

\section{Patient specimens}

We collected the CC and self-matched nearby normal cervical tissues from $50 \mathrm{CC}$ patients during the surgery treatment, and stored at $-80{ }^{\circ} \mathrm{C}$ after liquid nitrogen freezing.

\section{Informed consents}

The written informed consents were provided by all the $\mathrm{CC}$ patients before the tissues were collected. All experiments were agreed by the Ethics Committees of all participation hospitals following the Helsinki Declaration.

\section{Cell culture}

Four CC (SiHa, C-4I, HeLa and C-33A) and a normal cervical epithelial (End1/E6E7) cell lines were provided by the Committee on Type Culture Collection of the Chinese Academy of Sciences (Shanghai, China), which were maintained in the Macoy's 5A medium containing the streptomycin $(100 \mathrm{mg} / \mathrm{mL})$, penicillin $(100 \mathrm{IU} / \mathrm{mL})$ and FBS $(10 \%)$, and a humidified $5 \% \mathrm{CO}_{2}$ incubator at $37^{\circ} \mathrm{C}$.

\section{Reagents}

Propidium iodide, APC-Annexin V and protease inhibitor cocktail were provided by Sigma (St. Louis, MO, USA); CCK-8 test kit was provided by Dojindo Corp (Kyushu, Japan); lipofectamine 3000 and Trizol were from Thermo Fisher Scientific (Waltham, MA, USA); Apoptosis Detection Kit was from Beyotime (Nanjing, China); Dual-Luciferase Reporter Assay System was provided by Promega (Madison, WI, USA); matrigel was from BD (New Jersey, USA); Macoy's 5A medium was from Gibco (Rockford, MD, USA); SurePrep ${ }^{\mathrm{TM}}$ Nuclear or Cytoplasmic RNA Purification Kit was from Fisher BioReagents ${ }^{\circledR}$ (Fair Lawn, NJ, USA). Antibodies against the Bcl-2 (\# 3498), GAPDH (\#2118) and Cleaved caspase3 (\# 9661S) were obtained from Cell Signaling Technology, Inc. (Danvers, MA, USA); antibody against the DACT1 was from Novus Biologicals, LLC (Centennial, CO, USA).

\section{Bioinformatics analysis for target gene identification}

LncBASE was applied to predict the miRNAs potentially being sponged by H1FX-AS1. Targetscan was applied to predict the prospective binding sites of miR-324-3p with DACT1.

\section{Lentiviral vector, siRNA, inhibitors, mimics and luciferase activity assay}

The over-expression and control lentivirus vectors were bought from Hanbio (Shanghai, China).The genetically modified stable cells were established after the routine infection and selection following the manufacturer's instructions. SiRNA, mimics and the negative control (NC) were provided by GenePharma (Shanghai, China). Lipofectamine 3000 was used for target gene transfection following the manufacturer's instructions.

\section{Dual-luciferase reporter assay}

According to the bioinformatics prediction, miR-324-3p was designated as the potential target of H1FX-AS1, which could potentially bind to DACT1. The pmirGLO Dual-Luciferase miR Target Expression Vector was then 
applied to verify the direct interaction between the candidate miR-324-3p and the H1FX-AS1 (or DACT1). The wild-type (WT) reporter construct pmirGLO/H1FXAS1 (or DACT1) and the mutant reporter construct pmirGLO/H1FX-AS1-mut (or DACT1-mut) was cotransfected with miR-324-3p mimic or miR-NC (negative control) in $\mathrm{SiHa}$ and HeLa cells. The Firefly luciferase levels were checked by the microplate reader (a luminometer) $48 \mathrm{~h}$ after the transfection, and normalized to the Renilla luciferase activity, which was used as the final luciferase activity of the tested report gene.

\section{Cytoplasmic and nuclear RNA fraction isolation}

A SurePrep ${ }^{\mathrm{TM}}$ Nuclear or Cytoplasmic RNA Purification Kit (Cat. \# BP2805-25) was used to isolate the nuclear and cytoplasmic RNA fractions, following the manufacture's protocol. Briefly, the monolayer $\mathrm{SiHa}$ and HeLa cells were collected respectively and lysed using the Lysis Solution. The lysate was centrifuged for $3 \mathrm{~min}$ at 12,000 RPM. The supernatant containing the cytoplasmic RNA was transferred into an RNase-free tube, and retained the pellet containing the nuclear RNA, followed by binding the cytoplasmic and nuclear RNAs to the columns respectively. Then the columns were washed and RNAs were eluted. The cellular sublocalization expression of H1FX-AS1 was tested with RT-qPCR. U6 and GAPDH were used as the internal control.

\section{Total RNA isolation and RT-qPCR analysis}

The purity and concentration of the total RNA isolated with Trizol were evaluated with ND-1000 model NanoDrop (Thermo Fisher Scientific, MA, USA). PrimeScrip $^{\text {TM }}$ RT Master Mix for reverse transcription, SYBR Green Premix Ex $\mathrm{Taq}^{\mathrm{TM}}$ II for cDNA amplification and thermo recycler AB7300 (Applied Biosystems, CA, USA) with the TaqMan Universal PCR Master Mix for RTqPCR assay were performed respectively. The relative gene expression was represented by $2^{-\Delta \Delta C \mathrm{t}}$ method.

\section{Apoptosis evaluation}

The apoptosis was determined using the Apoptosis Detection Kit following the flow cytometric assay with the Cell Quest software (version 1.2.2, Becton Dickinson \& Company, Franklin Lakes, NJ, USA).

\section{Evaluation of colony formation ability}

The 7-day-cultured target cells, in a 6-well plate with the original concentration of 1000 cells/well, were fixed for $10 \mathrm{~min}$ in $4 \%$ paraformaldehyde, followed by staining for 5 min with $0.5 \%$ crystal violet. A light microscope (Olympus, Japan) and ImageJ were then applied for colony image and number collection.

\section{Cell viability evaluation}

The CCK-8 kit was used to evaluate the cell viability according to the manufacturer's instructions. Briefly, a $10 \mu \mathrm{L}$ of CCK- 8 reagent was added into the culture medium of cells to be tested in a 96-well plate, and cultured at $37^{\circ} \mathrm{C}$ in dark for $2 \mathrm{~h}$. The optional density (OD) was estimated at $450 \mathrm{~nm}$ using a 680 Microplate Reader (Bio-Rad, Hercules, CA).

\section{Wound healing assay for cell migration ability evaluation}

A $10-\mu$ l pipette tip was used to carefully scratch a wound at the middle of the wells of the 6-well plate with $100 \%$ confluent cells. The cells were washed with sterile PBS and then cultured in fresh serum-free medium at $37{ }^{\circ} \mathrm{C}$ for $24 \mathrm{~h}$ to get the cell migration images at 0 and $24 \mathrm{~h}$ under an inverted light microscope (Olympus, Japan).

\section{Transwell invasiveness ability assay}

A 24-well transwell with $8.0 \mu \mathrm{m}$ pore size (Corning Costar, USA) and coated Matrigel was used to estimate the invasiveness ability. In brief, $500 \mu \mathrm{L}$ of medium with $10 \%$ FBS was loaded into the lower chamber; $3 \times 10^{5}$ cells in $200 \mu \mathrm{l}$ of serum-free medium was loaded into the upper chamber for $48 \mathrm{~h}$ culture; the remained cells on the opposite side of the upper chamber after removing those on the upper side using a cotton swab, which represented the invaded cells, were fixed in $4 \%$ paraformaldehyde followed by $0.1 \%$ crystal violet staining.

\section{Total protein isolation and western blot assay} Cells to be tested were lysed with the NP40 lysis buffer (10\% glycerol, 5 mM EDTA, 0.1\% NP-40, $150 \mathrm{mM} \mathrm{NaCl}$ and $50 \mathrm{mM}$ Tris- $\mathrm{HCl}$ ) containing the protease inhibitor cocktail. Same amounts of proteins were separated on a $8 \%$ SDS-PAGE gel, transferred onto the PVDF membranes and immunoblotted with primary antibodies at $4{ }^{\circ} \mathrm{C}$ overnight, followed by secondary antibody culture at room temperature for $1 \mathrm{~h}$.

Antibodies against the Bcl-2(1:1000),GAPDH (1:1000) and Cleaved caspase 3 (1:1000); and antibody against the DACT1 (1:1000) were used.

\section{Animal experiments}

Forty female 6-week-old BALB/c nude mice were provided by Beijing HFK Bioscience Co. Ltd. (Beijing, China), divided into four groups $(\mathrm{n}=10)$ and housed in the animal room of Nantong University. All animal experimental processes were approved by the Animal Experimental Ethics Committee of Qidong Hospital Affiliated to Nantong University. The $\mathrm{SiHa}$ and HeLa cells over-expressed H1FX-AS1 or the NC $\left(1 \times 10^{7}\right.$ 
cells/mouse/200 $\mu \mathrm{l}$ of PBS) were injected subcutaneously on the back of the mice. The tumor volume was measured every week with a vernier caliper. Nude mice were sacrificed 35 days after inoculation, and the tumor weight was measured.

\section{Statistics process}

The data were presented as mean \pm SD. Two or multiple group comparison was performed by Student's t-test or one-way ANOVA respectively with the SPSS 20.0 (IBM, Chicago, USA). Survival curves were achieved by Kaplan-Meier analysis and compared with log-rank test. The relationship between genes was evaluated by Pearson Chi square analysis. A $p<0.05$ was statistically significant.

\section{Results}

Down-regulated H1FX-AS1 and up-regulated miR-324-3p were identified in both CC patients and cells, and low H1FX-AS1 expression was correlated with poor prognosis of CC patients

To explore the expression profile and the clinical function of H1FX-AS1 in CC patients, we analyzed the Cancer Genome Atlas (TCGA) datasets, and the results demonstrated that H1FX-AS1 expression was statistically significantly lower in the CC $(\mathrm{T})$ tissues than in the normal (N) tissues from the TCGA datasets (Fig. 1a, $p<0.05$ ), and analysis by GEPIA confirmed that the CC patients with low H1FX-AS1 expression level had a poor prognosis (Fig. $1 \mathrm{~b}, p<0.05$ ). To further verify the above results, we collected the CC and the self-matched adjacent normal cervical tissues, as well as the clinicopathological characteristics (Table 1) from 50 CC patients, as we can see in Fig. 1c, H1FX-AS1 expression level was statistically significantly lower in the $C C$ tissues than in the paired normal adjacent tissues analyzed by RT-qPCR $(p<0.01)$; after the $50 \mathrm{CC}$ patients were divided into the H1FX-AS1 low expression level group $(n=25)$ and the H1FX-AS1 high expression level group $(n=25)$ with the cut-off value of H1FX-AS1 median expression level in CC tissues, the survival curves of the CC patients with H1FX-AS1 high expression level and H1FX-AS1 low expression level were plotted by Kaplan-Meier analysis, which showed a poor prognosis in CC patients with H1FX-AS1 low expression level; the relationship between H1FX-AS1 expression level and the clinicopathological characteristics of $\mathrm{CC}$ patients analyzed by Chi square test showed that the H1FX-AS1 expression was statistically significantly related to the tumor size, the TNM stage, and the lymph node metastasis $(\mathrm{p}<0.05)$, while not related to the patient age and the tumor differentiation (Table 1 and Fig. 1d). The down-regulated H1FX-AS1 expression level was also verified in CC cells, as shown in Fig. 1e, the expression level of H1FX-AS1 was statistically significantly lower in all tested CC cells (SiHa, C-4I, HeLa and C-33A) versus the End1/E6E7 cells $(p<0.01)$. Meanwhile, the miR324-3p expression level was detected by RT-qPCR in both the CC tissues and cells, our results showed that miR-324-3p expression level was statistically significantly higher in the $\mathrm{CC}$ tissues than in the paired normal adjacent tissues from $50 \mathrm{CC}$ patients (Fig. 1f, $p<0.01$ ); miR324-3p expression level was statistically significantly higher in the CC cells (SiHa, C-4I, HeLa and C-33A) than in the normal human cervical epithelial End1/E6E7 cells (Fig. 1g, $p<0.01$ ). In additional, Pearson correlation analysis demonstrated that miR-324-3p showed a negative correlation with H1FX-AS1 expression level in the CC tissues from the $50 \mathrm{CC}$ patients (Fig. $1 \mathrm{~h}, p<0.001$ ). These results showed that H1FX-AS1 was down-regulated and miR-324-3p was up-regulated in both the CC patients and cells, down-regulated H1FX-AS1 expression level was correlated with the poor prognosis of CC patients.

\section{Over-expression of H1FX-AS1 inhibited proliferation, migration, and invasion, while induced apoptosis in CC cells}

The successful over-expression of H1FX-AS1, in the SiHa and HeLa cells with the lowest H1FX-AS1 expression, was determined by RT-qPCR analysis (Fig. 2a, p $<0.01$ ). We identified that over-expression of H1FX-AS1 significantly reduced the viability tested by the CCK- 8 assay

\footnotetext{
(See figure on next page.)

Fig. 1 H1FX-AS1 expression was significantly down-regulated and miR-324-3p was significantly up-regulated in CC tissues and cell lines. a H1FX-AS1 expression was statistically significantly lower in the CC (T) tissues than in the normal (N) tissues obtained by analyzing the TCGA datasets; b CC patients with low H1FX-AS1 expression had a poor prognosis from the TCGA datasets analyzed by GEPIA; $\mathbf{c}$ H1FX-AS1 expression was statistically significantly lower in the CC tissues than in the paired normal adjacent tissues from 50 CC patients analyzed by RT-qPCR; $\mathbf{d}$ the survival curve of the 50 CC patients with H1FX-AS1 high or low expression by KM-plotter plots, indicating poor prognosis in CC patients with H1FX-AS1 low expression; e H1FX-AS1 expression was statistically significantly lower in the CC cells (SiHa, C-4I, HeLa and C-33A) than in the normal human cervical epithelial End1/E6E7 cells analyzed by RT-qPCR, and the difference was most significant in the SiHa and the HeLa cells; $\mathbf{f}$ miR-324-3p expression was statistically significantly higher in the CC tissues than in the paired normal adjoining tissues from 50 CC patients analyzed by RT-qPCR; $\mathbf{g}$ miR-324-3p expression was statistically significantly higher in the CC cells (SiHa, C-4l, HeLa and C-33A) than in the normal human cervical epithelial End1/E6E7 cells analyzed by RT-qPCR; $\mathbf{h}$ Pearson correlation analysis of the association between miR-324-3p with H1FX-AS1 in the CC tissues from the 50 CC patients (same samples as in $\mathbf{c}$, and $\mathbf{f}$ ). ${ }^{* *} p<0.01$
} 

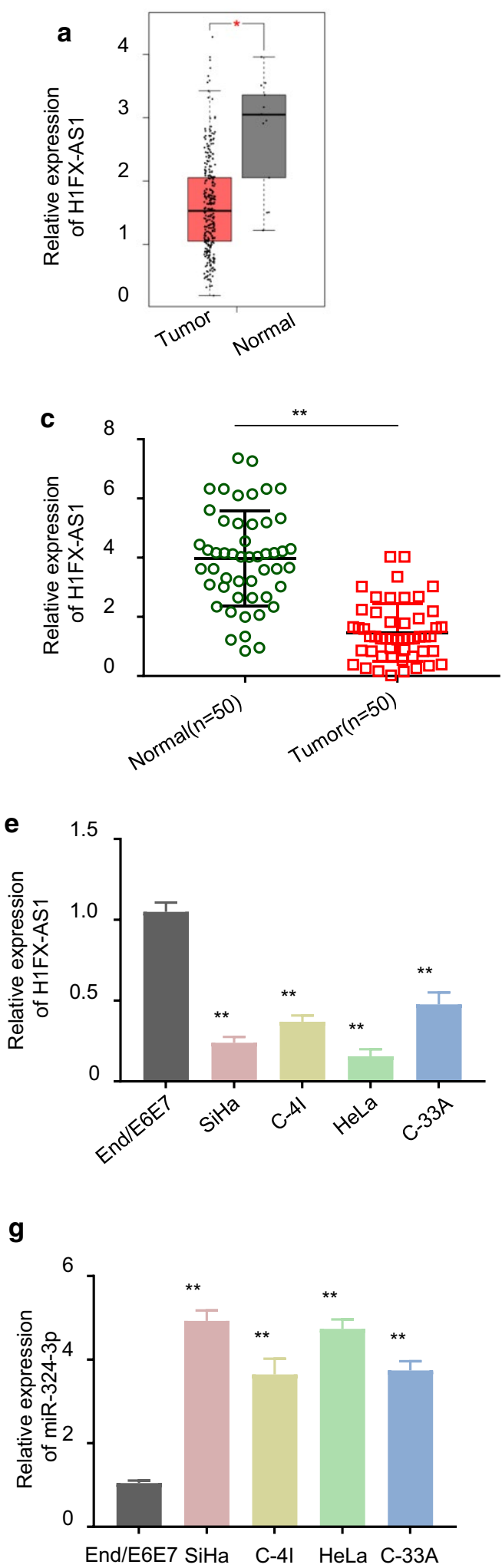

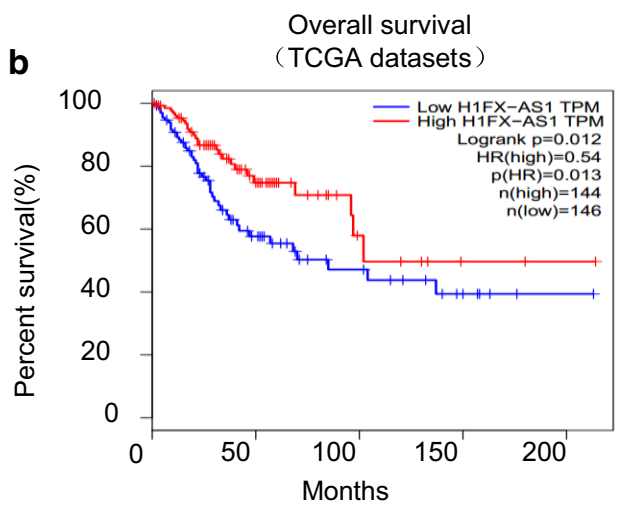

d
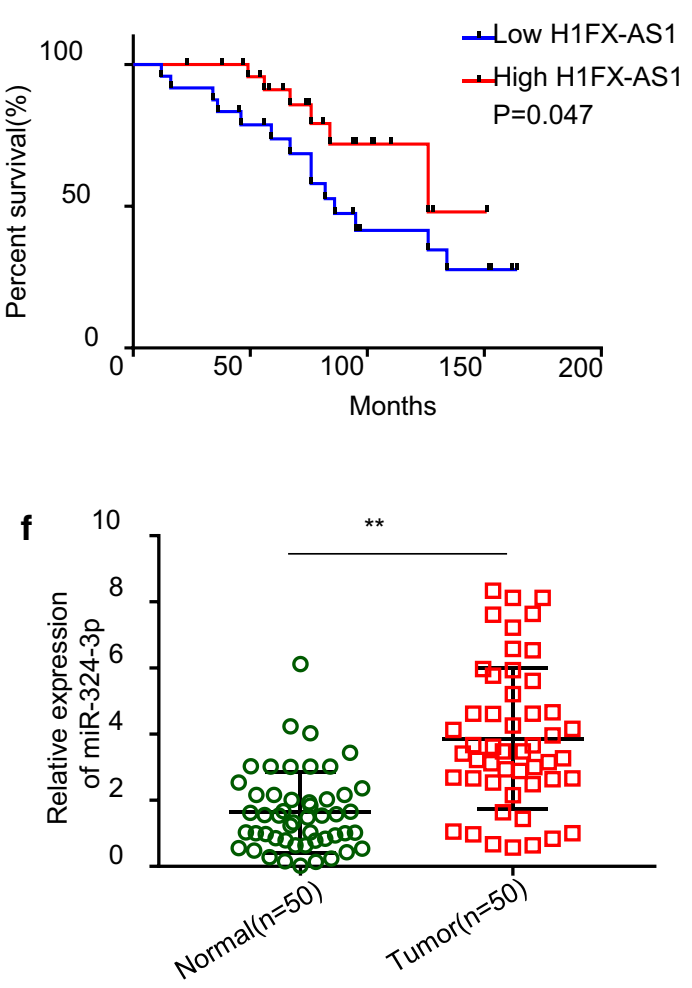

h

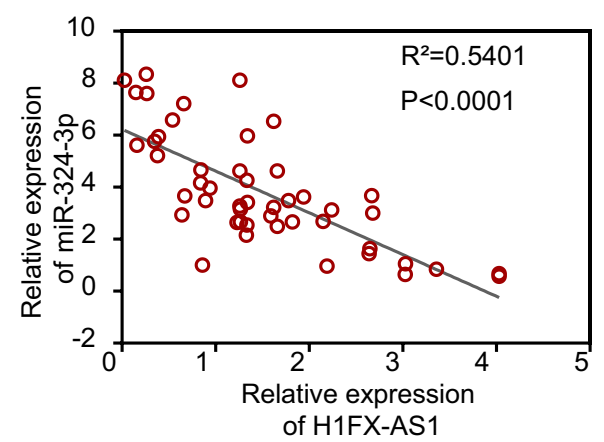


Table 1 The relationship between H1FX-AS1 expression and the clinicopathological characteristics of CC patients was analyzed by Chi square test

\begin{tabular}{|c|c|c|c|c|c|}
\hline Clinicopathological characteristics & Total & High expression & Low expression & $x^{2}$ & $P$ value \\
\hline \multicolumn{6}{|l|}{ Age } \\
\hline$\leq 35$ & 28 & 12 & 16 & 1.299 & 0.254 \\
\hline$>35$ & 22 & 13 & 9 & & \\
\hline \multicolumn{6}{|l|}{ Differentiation } \\
\hline High & 17 & 8 & 9 & 0.111 & 0.946 \\
\hline Moderate & 14 & 7 & 7 & & \\
\hline Poor & 19 & 10 & 9 & & \\
\hline \multicolumn{6}{|l|}{ Tumor size (cm) } \\
\hline$<4$ & 28 & 18 & 10 & 5.195 & 0.023 \\
\hline$\geq 4$ & 22 & 7 & 15 & & \\
\hline \multicolumn{6}{|l|}{ TMN stages } \\
\hline 1 & 15 & 12 & 3 & 14.274 & 0.003 \\
\hline$\|$ & 11 & 7 & 4 & & \\
\hline III & 13 & 5 & 8 & & \\
\hline IV & 11 & 1 & 10 & & \\
\hline \multicolumn{6}{|l|}{ Lymph node metastasis } \\
\hline Positive & 21 & 6 & 15 & 6.650 & 0.010 \\
\hline Negative & 29 & 19 & 10 & & \\
\hline
\end{tabular}

The 50 CC patients were divided into H1FX-AS1 low expression group $(n=25)$ and H1FX-AS1 high expression group $(n=25)$ with the cut-off value of H1FX-AS1 median expression in CC tissues

(Fig. 2b), the clone formation ability (Fig. 2c), cell migration tested by the wound healing assay (Fig. 2d) and invasive potential tested by the transwell analysis (Fig. 2e), while induced apoptosis tested by the flow cytometric analysis (Fig. 2f). In addition, the expression level of cleaved caspase 3 (the active apoptotic effector protein) was increased; while the anti-apoptotic protein Bcl-2 was decreased by over-expression of H1FX-AS1 in these two cell lines detected by western blot assay (Fig. 2g). A xenograft tumor model was then made to further confirm the effect on tumor growth after subcutaneous inoculation with H1FX-AS1 stably over-expressed SiHa or HeLa cells. We demonstrated that the tumor proliferative activity, including the tumor volume and tumor weight, was decreased in H1FX-AS1 over-expressed group versus the control vector group (Fig. $2 \mathrm{~h}, p<0.01$ ). Collectively, our results demonstrated that H1FX-AS1 acted as a tumorsuppressor to inhibit the tumorigenesis of CC.

\section{H1FX-AS1 served as a competing endogenous RNA to sponge miR-324-3p in CC cells}

Emerging evidences have reported that cytoplasmic lncRNAs predominantly serve as the competing endogenous RNAs (ceRNAs) through sponging the specific miRNAs that degrade the target genes [13, 14]. Given that H1FX-AS1 is a novel identified lncRNA, we performed a nuclear and cytoplasmic separation followed by RT-qPCR assay to determine the cellular sublocalization expression level of H1FX-AS1 in SiHa and HeLa cells. The results showed that the cytoplasmic H1FX-AS1 was predominant versus the nuclear fraction (Fig. 3a, $p<0.01)$, therefore, we hypothesized that H1FX-AS1

(See figure on next page.)

Fig. 2 Over-expression of H1FX-AS1 inhibited proliferation, migration and invasion, while induced apoptosis in CC cells both in vivo and in vitro. To investigate the influence of H1FX-AS1 expression in CC development, H1FX-AS1 was over-expressed in SiHa and HeLa cells (the two tested cell lines showing the lowest H1FX-AS1 expression), when RT-qPCR analysis confirmed that H1FX-AS1 was successfully over-expressed in both the SiHa and HeLa cells a, the following phenotypes were further estimated in the SiHa and HeLa cells over-expressed H1FX-AS1 (OE-H1FX-AS1): b cell viability, c clone formation ability (images: upper panel; quantification: lower panel), $\mathbf{d}$ cell migration(images: upper panel; quantification: lower panel), e cell invasion(images: left panel; quantification: right panel), $\mathbf{f}$ apoptosis (images: upper panel; quantification: lower panel), $\mathbf{g}$ apoptosis-related proteins (images: upper panel; quantification: lower panel). OE-H1FX-AS1 in both the SiHa and HeLa cells inhibited the xenograft tumor growth: $\mathbf{h}$ growth curve (tumor volume) analysis of the xenograft tumors with H1FX-AS1 over-expressed or the control vector transfected SiHa or HeLa cells; $\mathbf{i}$ the average tumor weights between the over-expressed or the control vector transfected SiHa or HeLa cell groups. ${ }^{* *} p<0.01$ 

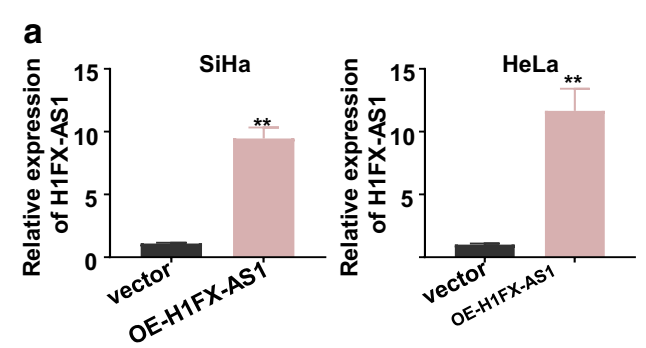

c

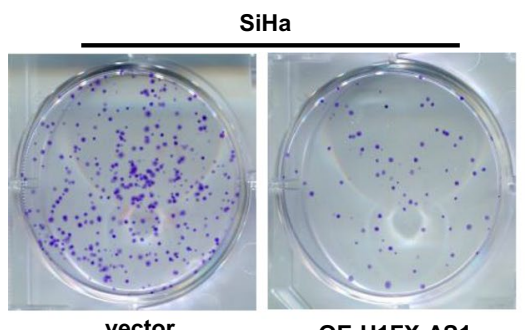

vector

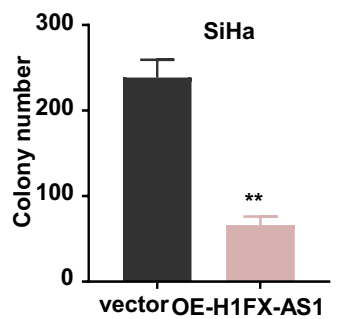

d

oh

$24 h$

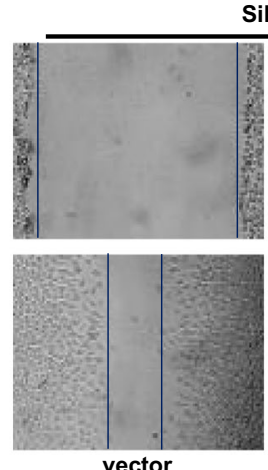

vector

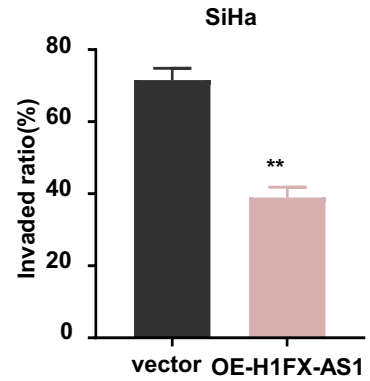

\section{b}
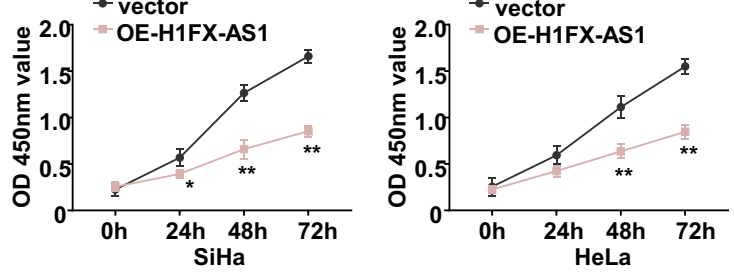

HeLa
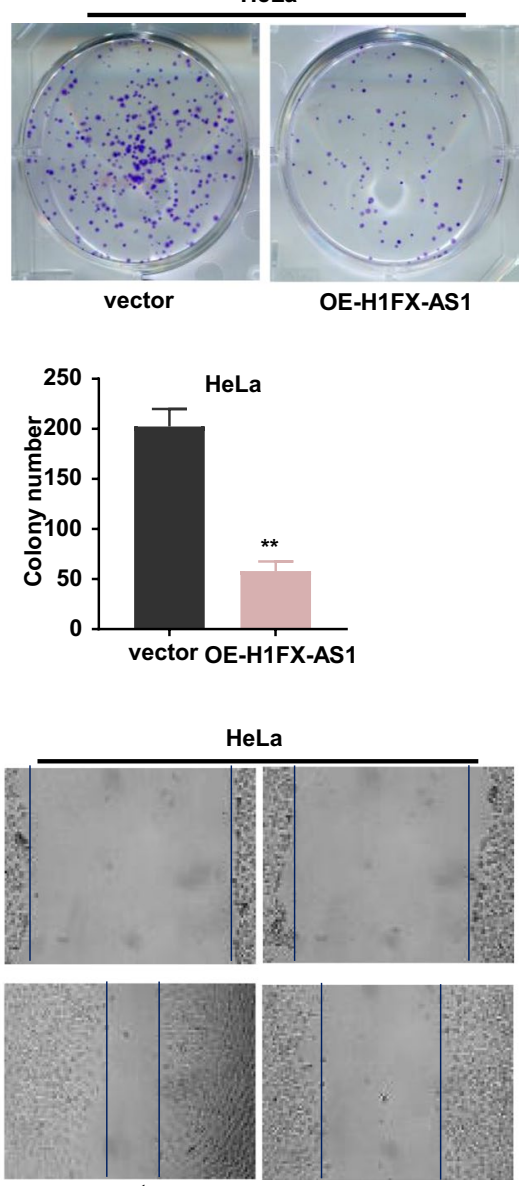

vector

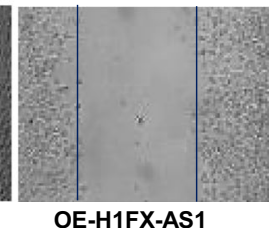

HeLa

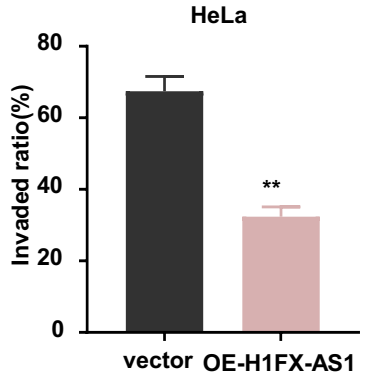




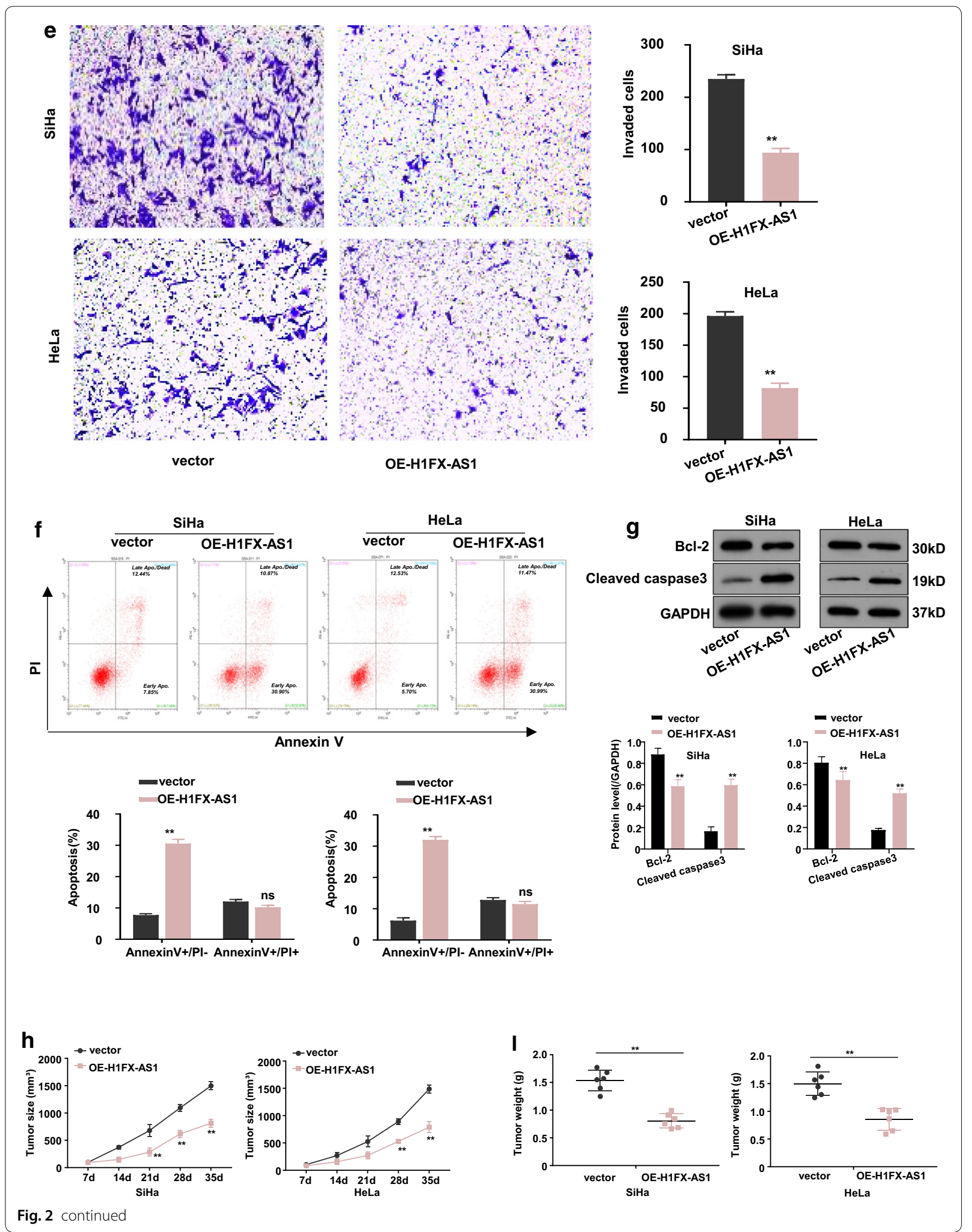


may be a sponge of miRNAs to rescue the expression of genes targeted by miRNAs in CC development. Herein, we investigated the potential miRNAs that could bind to H1FX-AS1 in CC cells. After the bioinformatics prediction by searching the lncBASE (http://carolina.imis.athen a-innovation.gr/diana_tools/web/index.php? $r=\operatorname{lncba}$ sev2\%2Findex-predicted), miR-324-3p was found to be potentially bound to H1FX-AS1, and the schematic representation of the predicted binding sites of H1FX-AS1 with miR-324-3p was shown in Fig. 3b. To verify the true interaction between H1FX-AS1 and miR-324-3p, we subcloned the H1FX-AS1, with WT or mutated reporter gene, into the pmirGLO dual luciferase reporter vector. Dual luciferase assay showed that over-expression of miR-324-3p with the mimics inhibited the luciferase activities of the wild-type H1FX-AS1 reporter gene in both the SiHa and HeLa cells $(\mathrm{p}<0.01)$, while this effect was neutralized after the predicted binding sites between H1FX-AS1 and miR-324-3p were mutated (Fig. 3c). The direct binding between H1FX-AS1 and miR-324-3p was further confirmed using RNA in vivo precipitation (RIP), which showed that, versus the control oligo probe, more miR-324-3p was statistically significantly pulled down by the H1FX-AS1 probe in both the SiHa and HeLa cells (Fig. 3d, $p<0.01$ ). Meanwhile, we found that H1FX-AS1 over-expression down-regulated miR-324-3p expression by over $50 \%$ in both the SiHa and HeLa cells $(p<0.01$, Fig. 3e). These results showed that the cytoplasmic H1FX-AS1 served as a ceRNA to sponge the miR-324-3p in CC cells.

H1FX-AS1 sponged miR-324-3p to up-regulate dishevelled binding antagonist of beta catenin 1(DACT1) in CC cells

DACT1 is a Wnt-pathway inhibitor, it has been reported that miR-324-3p activated Wnt/ $\beta$-catenin signaling via targeting DACT1 to promote hepatocellular carcinoma growth [15], meanwhile, we predicted the binding sites of miR-324-3p in the $3^{\prime}$ non-coding region(UTR) of DACT1 by targetscan (Fig. 4). Therefore, we hypothesized that miR-324-3p may directly bind to and inhibit the DACT1 expression. We then subcloned the DACT1 reporter gene with WT or mutated 3 UTR of DACT1 into the pmirGLO dual luciferase reporter vector. Dual luciferase assay displayed that over-expression of miR-324-3p with the mimics inhibited the luciferase activity of the wild-type DACT1 reporter gene in both the SiHa and HeLa cells $(p<0.01)$, while this effect was diminished after the predicted binding sites between miR-324-3p and the 3'UTR of DACT1 were mutated (Fig. 4b), indicating the existence of the direct interaction between the miR324-3p and the 3'UTR of DACT1. This direct interaction was further confirmed by measure the mRNA and protein expression levels of DACT1 in the SiHa and HeLa cells after over-expressing or silencing miR324-3p, which showed that knockdown (miR-324-3p inh) or over-expression (miR-324-3p mimics) of miR324-3p respectively increased or decreased the mRNA (Fig. 4c, $p<0.01$ ) and protein (Fig. 4 d, $p<0.01$ ) expression levels of DACT1. Since H1FX-AS1 competes the binding to the $3^{\prime} \mathrm{UTR}$ of DACT1 with miR-324-3p, we wondered whether H1FX-AS1 modulates miR-324-3pmediated inhibition of DACT1. To find out whether H1FX-AS1 sponges miR-324-3p, thus to regulate DACT1 expression in $\mathrm{SiHa}$ and HeLa cells, we first detected the mRNA and the protein expression levels of DACT1 in the SiHa and HeLa cells after H1FX-AS1 over-expression with or without miR-324-3p co-transfection by RT-qPCR and Western blot assays, our results revealed that H1FX-AS1 over-expression significantly up-regulated DACT1 expression at both the mRNA (Fig. 4e, $p<0.01$ ) and protein (Fig. 4f, $p<0.01$ ) levels in the SiHa and HeLa cells, and these effects were sufficiently reversed by co-transfection of the miR324-3p mimics. DACT1 expression level was further quantified with RT-qPCR in the CC tissues from $50 \mathrm{CC}$ patients, which proved that DACT1 expression was significantly decreased in the CC tissues versus the nearby normal tissues (Fig. 4g, $p<0.01$ ). Meanwhile, DACT1 expression level in the CC tissues from $50 \mathrm{CC}$ patients was found to be statistically significantly and positively related to the H1FX-AS1 expression level (Fig. 4h, the left panel, $p<0.001$ ), while statistically significantly and negatively related to the miR-324-3p expression level by the Pearson correlation analysis (Fig. $4 \mathrm{~h}$, the right

\footnotetext{
(See figure on next page.)

Fig. 3 H1FX-AS1 acted as a sponge for miR-324-3p. a Cellular sublocalization of the H1FX-AS1 in the SiHa and HeLa cells were determined by the nuclear and cytoplasmic separation experiments, U6 and GAPDH are used as internal references for nuclear and cytoplasmic expression, respectively. The potential binding sites of H1FX-AS1 with miR-324-3p were identified: the schematic representation of the potential binding sites of H1FX-AS1 with miR-324-3p predicted by InCBASE (b), which was verified by double luciferase reporter assay in the SiHa (left panel) and HeLa (right panel) cells after co-transfection of H1FX-AS1 WT or mutated reporter with or without miR-324-3p mimics (c); miR-324-3p in the cell lysates of $\mathrm{SiHa}$ (left panel) and HeLa (right panel) cells was further pulled down and enriched with biotin labeled H1FX-AS1 specific probe (d); the miR-324-3p expression level was confirmed to be negatively related with the H1FX-AS1 expression level after checking the miR-324-3p expression level in the $\mathrm{SiHa}$ (left panel) and HeLa (right panel) cells with (OE-H1FX-AS1) or without (vector) H1FX-AS1 over-expression (e). ${ }^{* *} p<0.01$
} 
a

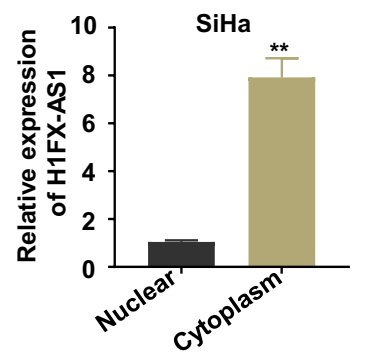

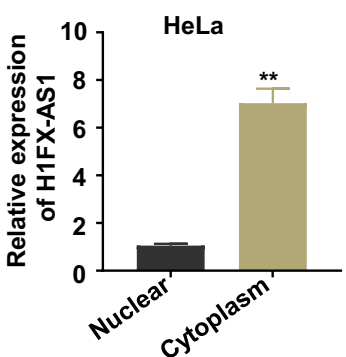

b

H1FX-AS1 3'UTR 168-186 H1FX-AS1 wt 5'-UCAUCUCCCCGUGUGGCACCUGGGGCAGC-3' miR-324-3p 3'-GGU--CGUCGUGGACCCCGUCA-5' H1FX-AS1 mut 5'-UCAUCUCCUUGUAUGUAGUUGUUUUAGUC-3'

c

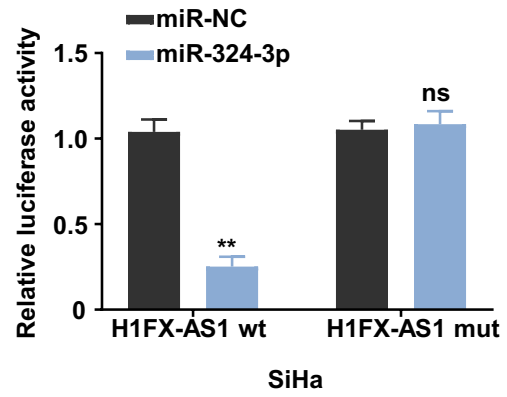

d

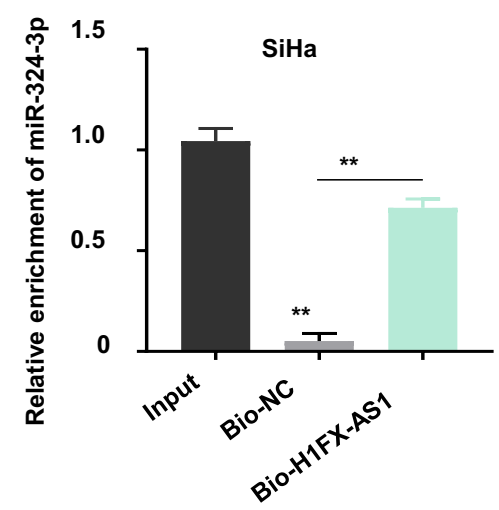

e

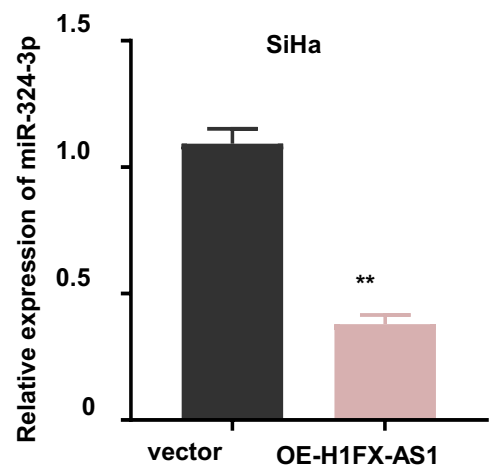

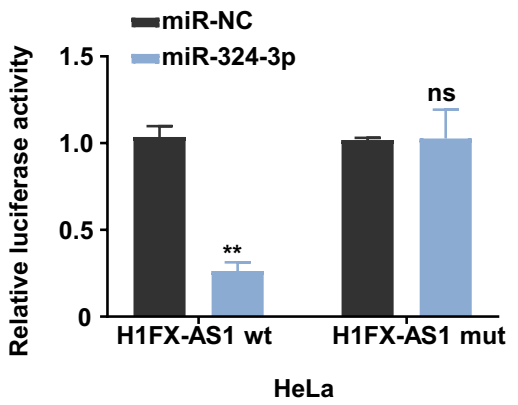
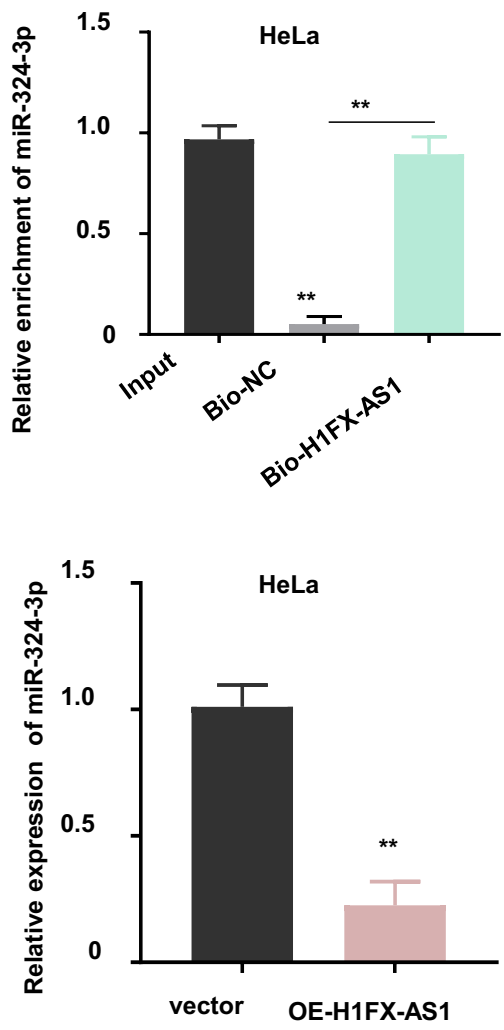
panel, $p<0.001)$. These results showed that DACT1 was up-regulated when the miR-324-3p was sponged by the H1FX-AS1 in the CC cells.

\section{The miR-324-3p over-expression or DACT1 knockdown blocked H1FX-AS1 induced inhibition of the aggressive behaviors in CC cells}

To further confirm the functional effect of the H1FXAS1/miR-324-3p/DACT1 axis in SiHa and HeLa cells, the cell viability, clone formation ability, apoptosis, apoptosis-related proteins, migration and invasion in $\mathrm{SiHa}$ and HeLa cells after H1FX-AS1 over-expression (OEH1FX-AS1) with or without miR-324-3p over-expression by miR-324-3p mimics (miR-324-3p) or DACT1 knockdown by small interfering RNA (si-DACT1) were further investigated, respectively. The results showed that over-expression of H1FX-AS1 statistically significantly reduced the OD values of the $\mathrm{SiHa}$ and HeLa cells at $450 \mathrm{~nm}$ wavelength, detected by CCK- 8 assay at different time points $(0 \mathrm{~h}, 24 \mathrm{~h}, 48 \mathrm{~h}$ and $72 \mathrm{~h})$, and this effect was partially rescued when the miR-324-3p mimics or si-DACT1 was co-transfected (Fig. $5 \mathrm{a}, \mathrm{p}<0.01$ ); the clone formation abilities of $\mathrm{SiHa}$ and HeLa cells were also statistically significantly reduced after H1FX-AS1 over-expression, which was partially rescued when the miR-324-3p mimics or si-DACT1 was co-transfected (Fig. 5b, p < 0.01); the apoptotic rates of SiHa and HeLa cells, detected by the flow cytometric analysis, were statistically significantly increased by over-expression of H1FX-AS1, while this effect was partially reduced when co-transfected with miR-324-3p mimics or siDACT1(Fig. 5c, p<0.01); the western blot assay showed that the increased expression of cleaved Caspase 3 and the decreased Bcl-2 expression after over-expression of H1FX-AS1 were partially rescued when the miR-324-3p mimics or si-DACT1 was co-transfected in $\mathrm{SiHa}$ and HeLa cells (Fig. 5d); the wound healing assay showed that the reduced cell migration abilities of $\mathrm{SiHa}$ and $\mathrm{HeLa}$ cells after over-expression of H1FX-AS1 were partially recovered when co-transfected with miR-324-3p mimics or si-DACT1 (Fig. 5e); the transwell assay showed that the reduced cell invasive abilities of $\mathrm{SiHa}$ and HeLa cells after over-expression of H1FX-AS1 were also statistically significantly reversed when co-transfected with the miR-324-3p mimics or si-DACT1 (Fig. 5f). Collectively, all of these observations indicate that H1FX-AS1 sponges miR-324-3p to activate DACT1, thus to inhibit the highly aggressiveness of the $\mathrm{CC}$ cells. These results showed that over-expressed miR-324-3p or inhibited DACT1 blocked the preventative effect of H1FX-AS1 in CC development.

\section{Discussion}

More than $80 \%$ of genes in the human genome are noncoding ones including the IncRNAs, which were originally believed to be the transcriptional "noise" and have been recently reported functional necessary molecules $[8$, 16].

In current study, we first identified H1FX-AS1, a novel lncRNA, was down-regulated in the CC cells and tissues, also the TCGA datasets, suggesting a prospective tumor suppressor gene in CC.

Kaplan-Meier analysis further confirmed that the low H1FX-AS1 expression indicated a poor prognosis in CC patients, which was significantly related to the tumor size, TNM stage, and lymph node metastasis, suggesting a potential prognostic marker or therapeutic target for CC patients.

The slight difference in the clinical survival rate between the TCGA dataset with our local patients may be caused by the varied regions where the patients came from. Patients in the TCGA database were global, while our local patients only included those from China. However, the results from these two sets showed the same pattern, thus we presented both in our current work to solidate the evidences of our conclusion.

Different molecular mechanisms can be applied to regulate the gene and protein functions since the cellular lncRNAs distribute in cytoplasm and/or nucleus, therefore, abnormal lncRNAs may trigger various cellular dysfunction, such as malignant transformation [17]. Our results found that H1FX-AS1 was a cytoplasmic lncRNA. Cumulative studies have reported that cytoplasm lncRNAs predominantly serve as ceRNAs by sponging the specific miRNAs and protect miRNAs

\footnotetext{
(See figure on next page.)

Fig. 4 H1FX-AS1 promoted DACT1 expression via sponging miR-324-3p. The schematic representation of the potential binding sites of miR-324-3p in the $3^{\prime}$ UTR of DACT1 predicted by targetscan (a), which was further confirmed by the dual-luciferase reporter assay of the SiHa (b, left panel) and HeLa (b, right panel) transfected by the WT or mutated DACT1-3' UTR reporter with or without co-transfection of miR-324-3p; the mRNA (c) and the protein (d, images: left panel; quantification: right panel) expression levels of DACT1 in the SiHa and HeLa cells after miR-324-3p was silenced (miR-324-3p mimics) or over-expressed (miR-324-3p mimics); the mRNA (e) and the protein (f, images: left panel; quantification: right panel) expression levels of DACT1 in the SiHa and HeLa cells after H1FX-AS1 over-expression with or without miR-324-3p co-transfection; $\mathbf{g}$ the relative expression levels of DACT1 in paired CC and nearby normal tissues from 50 patients (same samples as in Fig. 1C, f) detected by RT-qPCR; $\mathbf{h}$ Pearson correlation analysis of the associations between DACT1 with H1FX-AS1 (left panel) and miR-324-3p (right panel) in the CC tissues from 50 CC patients (same samples as in Fig. 1c, f). ${ }^{* *} p<0.01$
} 


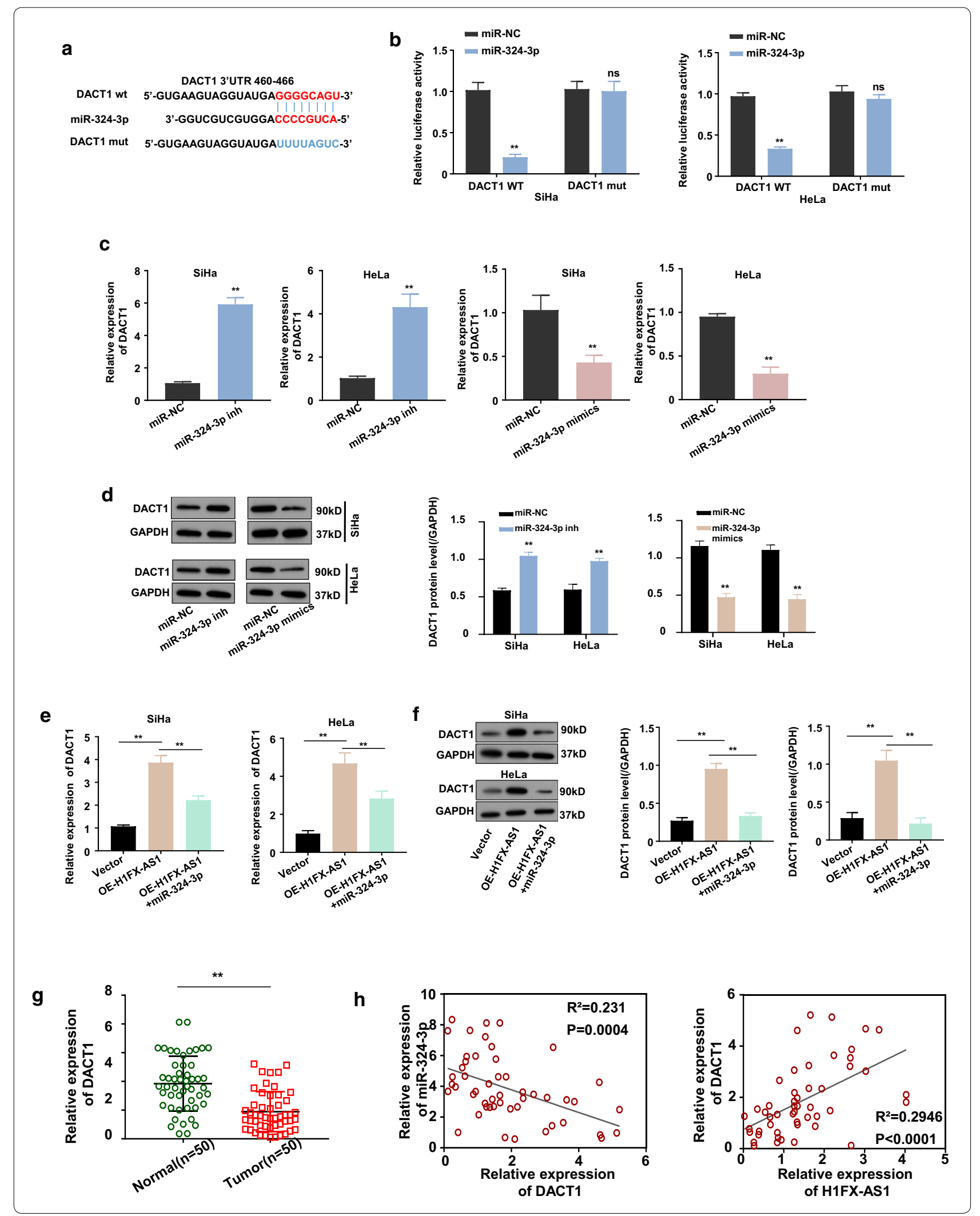


a

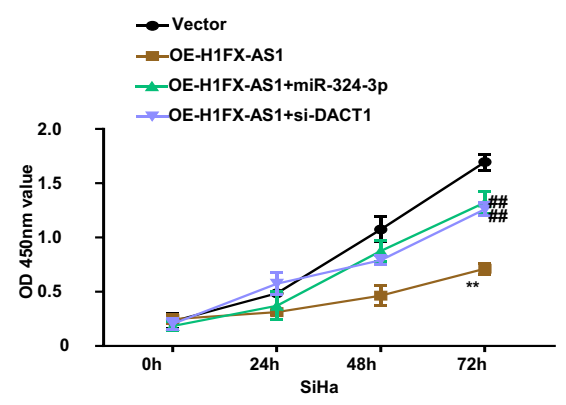

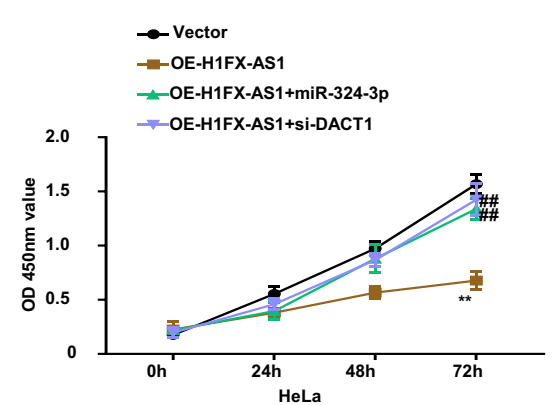

b
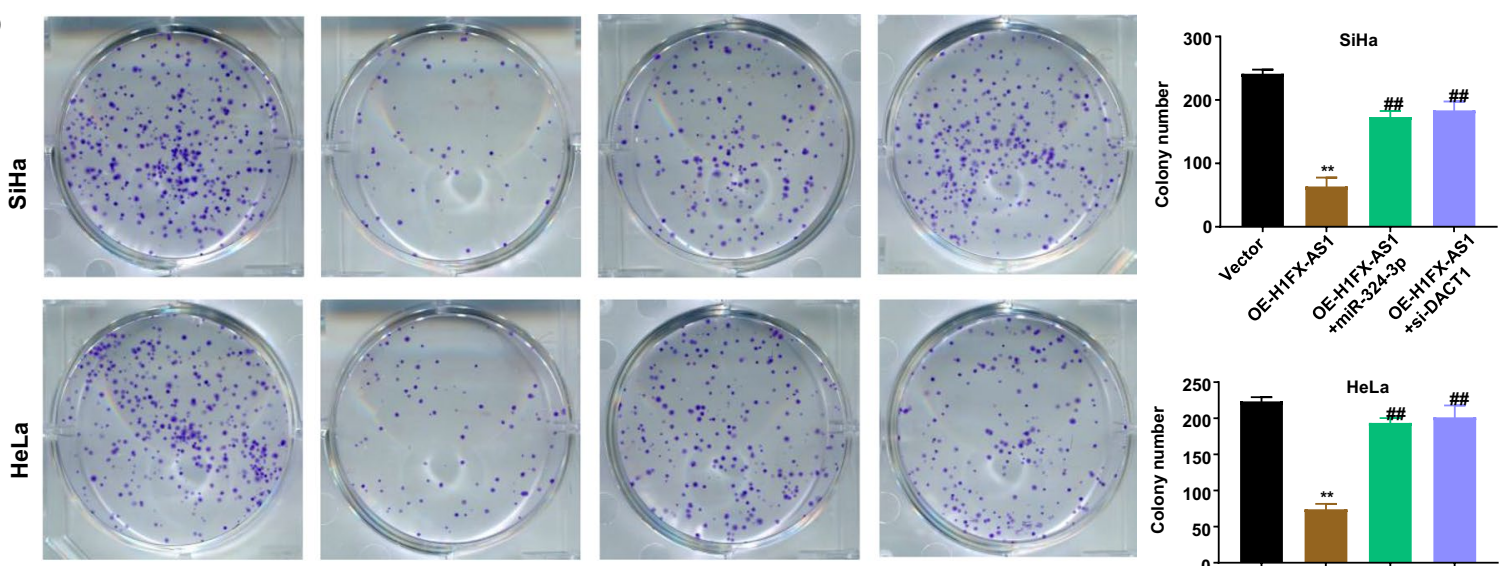

Vector

OE-H1FX-AS1

OE-H1FX-AS1

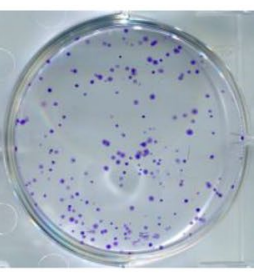

OE-H1FX-AS1 +si-DACT1

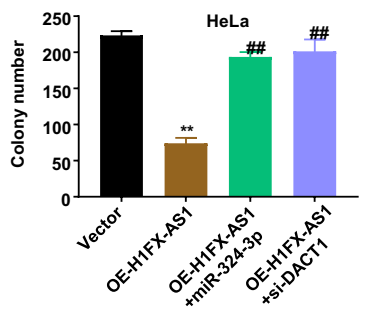

C Vector OE-H1FX-AS1 OE-H1FX-AS1
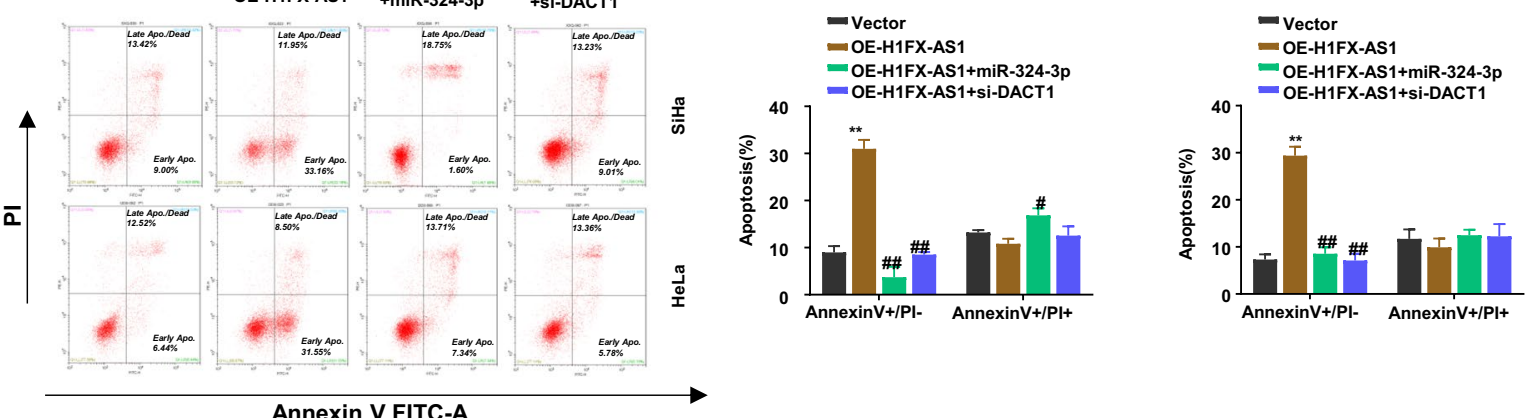

Annexin V FITC-A

\section{d}

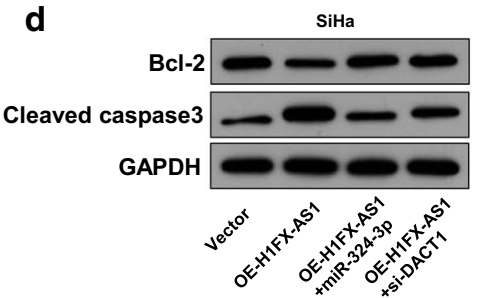

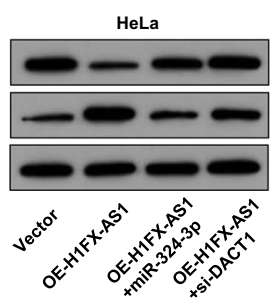
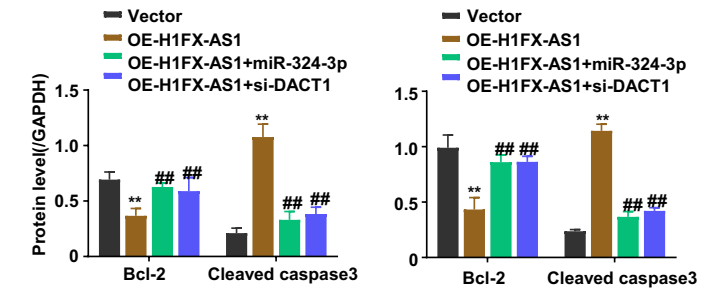

Fig. 5 continued 


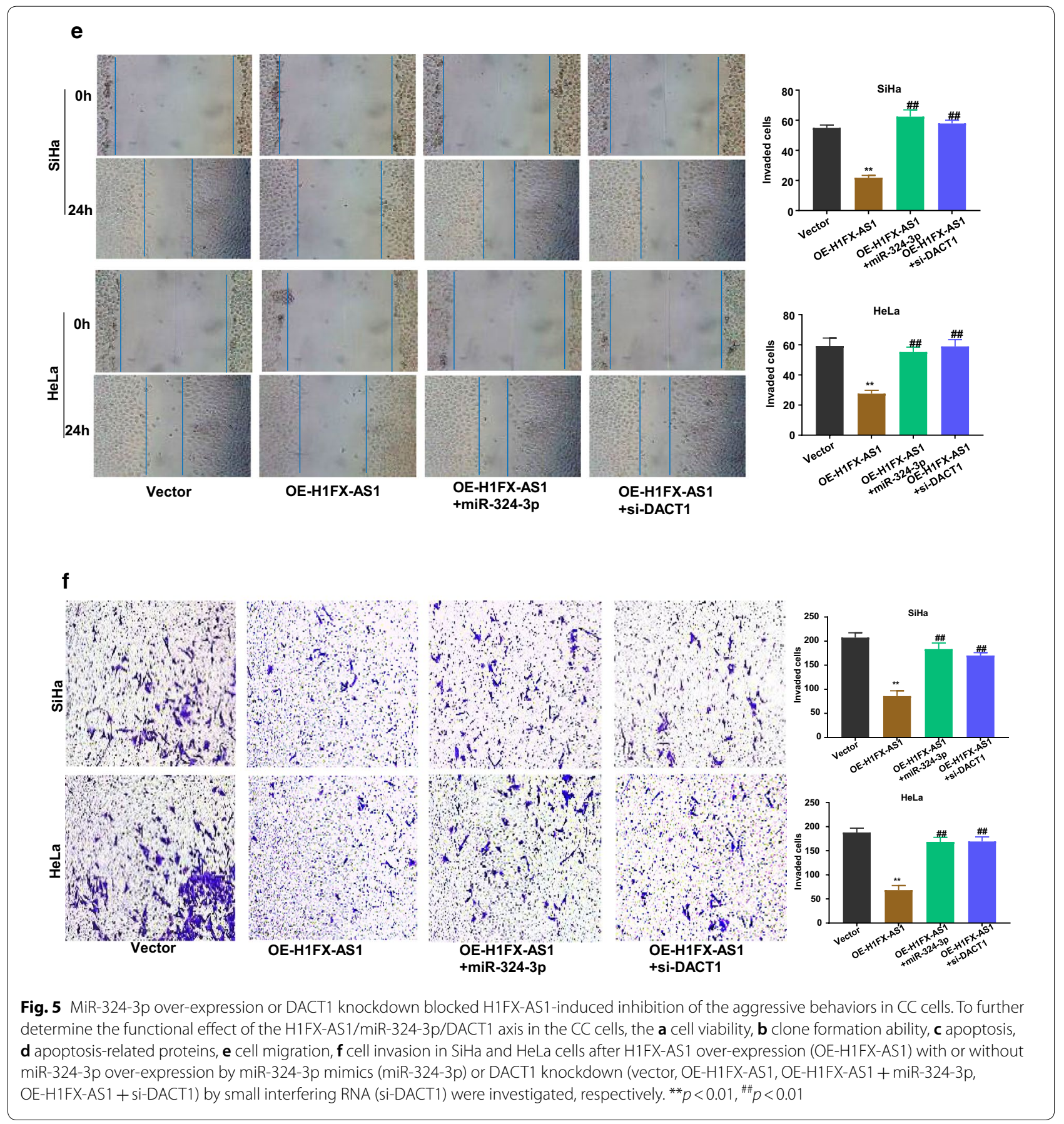

from binding to and degrading the target genes [1214]. Therefore, the competing miRNA being sponged by H1FX-AS1 and the target mRNA regulated by the miRNA were further explored. Bioinformatics analysis showed that miR-324-3p was the potential miRNA sponged by H1FX-AS1, which was further confirmed by Pearson correlation, dual luciferase reporter gene and RIP assays. Bioinformatics analysis and literature both revealed that DACT1 was the target gene of miR-324-3p, which was further verified by the Pearson correlation assay, and the function rescue experiments. Thus, in this study, we also revealed a novel mechanism that H1FXAS1 served as a ceRNA of miR-324-3p to up-regulate the DACT1 expression. Considering the important function of Wnt-signaling pathway in CC development and DACT1 is the inhibitor of the Wnt-signaling, our results for the first time found out the therapeutic significance of H1FX-AS1/miR-324-3p/DACT1 axis in CC patients. 


\section{Conclusion}

Our current study revealed that H1FX-AS1 expression was significantly down-regulated in $\mathrm{CC}$ patients and cells. Lower H1FX-AS1 expression in CC tissues was found to be associated with the poor prognosis of CC patients. Functionally, H1FX-AS1 inhibited CC cell growth, clone formation, migration and invasion, while induced apoptosis by sponging miR-324-3p to upregulate the DACT1 expression. Therefore, our findings highlighted the prospective role of H1FX-AS1 as a new prospective tumor suppressor gene and a prognostic predictor in CC patients, and a ceRNA of miR324-3p to inhibit DACT1 mediated CC progression. As a result, targeting H1FX-AS1/miR-324-3p/DACT1 axis would be a novel therapeutic strategy for CC.

\section{Acknowledgements}

We thank the reviewers for their constructive comments.

\section{Authors' contribution}

$\mathrm{HC}$ and WZ designed and directed the experiments; XS, JH and XG conducted the experiments and data analysis. All authors read and approved the final manuscript.

\section{Funding}

This study was supported by grants from (1) Hospital Scientific Research Fund (SDFEYGJ1708), (2) Jiangsu Province Maternal and Child Health Key Talent Project (FRC201714).

\section{Data available on request due to privacy/ethical restrictions}

All data supporting the results of this work are available from the corresponding author upon request. These data are not available in public because of the ethical restrictions or privacy.

\section{Consent for publication}

All authors consent to publication.

\section{Competing interests}

The authors declared they have no competing interests in this work.

\section{Author details \\ ${ }^{1}$ Department of Obstetrics and Gynecology, the Second Affiliated Hospital of Soochow University, No. 1055 Sanxiang Road, Suzhou 215000, China. ${ }^{2}$ Department of Obstetrics and Gynecology, Qidong People's Hospital of Jiangsu Province/Qidong Hospital Affiliated to Nantong University, No. 753 Jianghai Middle Road, Qidong 226200, China. ${ }^{3}$ Obstetrics and Gynecology Clinic, No. 963 Hospital, Jiamusi 154003, China. ${ }^{4}$ Obstetrics and Gynecology SAMII Medical Center in ShenZhen, No. 1, West Jinniu Road, Shenzhen 518118, China. ${ }^{5}$ Department of Gynecology, Maternal and Child Health Hospital of Liaocheng, No. 56 Changjiang Road, High-tech Zone, Liaocheng 252000, China.}

Received: 21 February 2020 Accepted: 26 June 2020 Published online: 31 July 2020

\section{References}

1. Bray F, Ferlay J, Soerjomataram I, Siegel RL, Torre LA, Jemal A. Global cancer statistics 2018: GLOBOCAN estimates of incidence and mortality worldwide for 36 cancers in 185 countries. CA Cancer J Clin. 2018;68(6):394-424.

2. Allemani C, Weir HK, Carreira H, Harewood R, Spika D, Wang XS, Bannon F, Ahn JV, Johnson CJ, Bonaventure A, et al. Global surveillance of cancer survival 1995-2009: analysis of individual data for 25,676,887 patients from 279 population-based registries in 67 countries (CONCORD-2). Lancet. 2015;385(9972):977-1010.

3. Wang BG, Xu Q, Lv Z, Fang XX, Ding HX, Wen J, Yuan Y. Association of twelve polymorphisms in three onco-IncRNA genes with hepatocellular cancer risk and prognosis: a case-control study. World J Gastroenterol. 2018;24(23):2482-90.

4. Zhao M, Wang S, Li Q, Ji Q, Guo P, Liu X. MALAT1: a long non-coding RNA highly associated with human cancers. Oncol Lett. 2018;16(1):19-26.

5. Batista PJ, Chang HY. Long noncoding RNAs: cellular address codes in development and disease. Cell. 2013;152(6):1298-307.

6. Wu H, Yang L, Chen LL. The diversity of long noncoding RNAs and their generation. Trends in genetics : TIG. 2017;33(8):540-52.

7. Guttman M, Russell P, Ingolia NT, Weissman JS, Lander ES. Ribosome profiling provides evidence that large noncoding RNAs do not encode proteins. Cell. 2013;154(1):240-51.

8. Schmitt AM, Chang HY. Long noncoding RNAs in cancer pathways. Cancer Cell. 2016;29(4):452-63.

9. Wapinski O, Chang HY. Long noncoding RNAs and human disease. Trends Cell Biol. 2011;21(6):354-61.

10. Flynn RA, Chang HY. Long noncoding RNAs in cell-fate programming and reprogramming. Cell Stem Cell. 2014;14(6):752-61.

11. Lu MH, Tang B, Zeng S, Hu CJ, Xie R, Wu YY, Wang SM, He FT, Yang SM. Long noncoding RNA BC032469, a novel competing endogenous RNA, upregulates hTERT expression by sponging miR-1207-5p and promotes proliferation in gastric cancer. Oncogene. 2016;35(27):3524-34.

12. Tay Y, Rinn J, Pandolfi PP. The multilayered complexity of ceRNA crosstalk and competition. Nature. 2014;505(7483):344-52.

13. Cao C, Zhang T, Zhang D, Xie L, Zou X, Lei L, Wu D, Liu L. The long noncoding RNA, SNHG6-003, functions as a competing endogenous RNA to promote the progression of hepatocellular carcinoma. Oncogene. 2017;36(8):1112-22

14. Qu L, Ding J, Chen C, Wu ZJ, Liu B, Gao Y, Chen W, Liu F, Sun W, Li XF, et al. Exosome-transmitted IncARSR promotes sunitinib resistance in renal cancer by acting as a competing endogenous RNA. Cancer Cell. 2016;29(5):653-68.

15. Tuo H, Wang Y, Wang L, Yao B, Li Q, Wang C, Liu Z, Han S, Yin G, Tu K, et al. MiR-324-3p promotes tumor growth through targeting DACT1 and activation of Wnt/beta-catenin pathway in hepatocellular carcinoma. Oncotarget. 2017;8(39):65687-98.

16. Louro R, Smirnova AS, Verjovski-Almeida S. Long intronic noncoding RNA transcription: expression noise or expression choice? Genomics. 2009:93(4):291-8.

17. Lalevee S, Feil R. Long noncoding RNAs in human disease: emerging mechanisms and therapeutic strategies. Epigenomics. 2015;7(6):877-9.

\section{Publisher's Note}

Springer Nature remains neutral with regard to jurisdictional claims in published maps and institutional affiliations.

Ready to submit your research? Choose BMC and benefit from

- fast, convenient online submission

- thorough peer review by experienced researchers in your field

- rapid publication on acceptance

- support for research data, including large and complex data types

- gold Open Access which fosters wider collaboration and increased citations

- maximum visibility for your research: over 100M website views per year

At BMC, research is always in progress.

Learn more biomedcentral.com/submissions 